\title{
Characterization of chemical coating on the functionalized carbon nanotubes
}

\author{
Z.P. Luo, ${ }^{*}$ R.L. Littleton, ${ }^{*}$ H. Kim, ${ }^{*}$ L. Adams, ${ }^{* *}$ L. Carson, ${ }^{* * *}$ N. Soboyejo, ${ }^{* *}$ and A. Oki ${ }^{* *}$ \\ * Microscopy and Imaging Center, Texas A\&M University, College Station, TX 77843-2257 \\ ** Department of Chemistry, Prairie View A\&M University, Prairie View, TX 77446 \\ *** Cooperative Agricultural Research Center, Prairie View A\&M University, Prairie View, TX \\ 77446
}

Carbon nanotubes (CNTs) have received considerable attention due to their extraordinary properties of strength, toughness, as well as thermal and electrical conductivities. They are ideal fillers for polymer nanocomposites to enhance the composite physical and mechanical properties. In order to overcome the problem of tangling caused by CNT intrinsic van der Waals forces during the composite fabrication, a chemical functionalization process was introduced [1-4] to achieve a better dispersion degree, which is a critical factor dominating the composite performance [5]. However, the coating microstructure remains unidentified. In this work, we present a characterization of the chemical coating on the functionalized CNTs using analytical TEM.

The experimental material, CNT-COCl, was oxidized with mixed sulfuric and nitric acids, and then further reacted with thionyl chloride $\mathrm{SOCl}_{2}$. The $\mathrm{CNT}$ sample was deposited on $\mathrm{Cu}$ grids coated with carbon support film, and observed in a FEI Tecnai $\mathrm{G}^{2} \mathrm{~F} 20$ at $200 \mathrm{kV}$, equipped with a Fischione ultra-high resolution STEM HAADF detector, Oxford instruments EDS detector, and a post-column Gatan Image Filter (GIF).

Fig. 1(a) is a typical TEM image of the functionalized CNTs, with coatings on the surface. From the Z-contrast image collected by the HAADF detector in the STEM mode, the coatings are more clearly revealed, as shown in (b). It is seen that most of the CNTs are coated with thick layers, while only a few areas have thin layers or are even uncoated. Particles with extremely high brightness, as indicated by arrowheads, are identified as metal particles of Co that give rise to high scattering contrast. The EDS analysis, as shown in (c), reveals the chemical compositions of C-S-Cl-O of the coating layers, consistent with the materials used during the functionalization process. The $\mathrm{Cu}$ signal originates from the grid bar.

In order to identify the chemical distribution, elemental mapping was performed by the threewindow method using GIF in the EFTEM mode. Fig. 2(a) is a zero-loss image, and the elemental maps of $\mathrm{O}, \mathrm{Cl}$ and $\mathrm{S}$ are shown in (b-d), respectively. Basically, all O-Cl-S elements distribute over the areas around the CNTs, while there are some small particles enriched in S, as highlighted by arrowheads in (d). A framed area in (a) is magnified in (e), with the carbon layers of the CNTs readily visible. Isolated surfactants discretely cover the vertical CNT, as indicated by arrowheads. The chemical coating provides an important role in facilitating excellent bonding with the polymer matrix [6].

References

[1] D.E. Hill, Y. Lin, A.M. Rao, L.F. Allard, Y.P. Sun. Macromol. 35 (2002) 9466.

[2] D. McIntosh, V.N. Khabashesku, E.V. Barrera. J. Phys. Chem. 111C (2007) 1592.

[3] C.H. Tseng, C.C. Wang, C.Y. Chen. Chem. Mater. 19 (2007) 308. 
[4] A. Oki, L. Adams, V. Khabashesku, Y. Edigin, P. Biney, Z.P. Luo. Mater. Lett. 62 (2008) 918.

[5] Z.P. Luo, J.H. Koo. J. Microsc. 225 (2007) 118; Polymer 49 (2008) 1841; Mater. Lett. 62 (2008) 3493.

[6] The authors thank NIH-NIAMSD Grant \# ARI49172, NIH-NIGMS RISE Grant \#1 R25 GM078361-01, the Welch Foundation, the US Air Force Research Laboratory, and TAMU VPR Office for supporting this work, and Dr. A. Holzenburg for his encouragement.
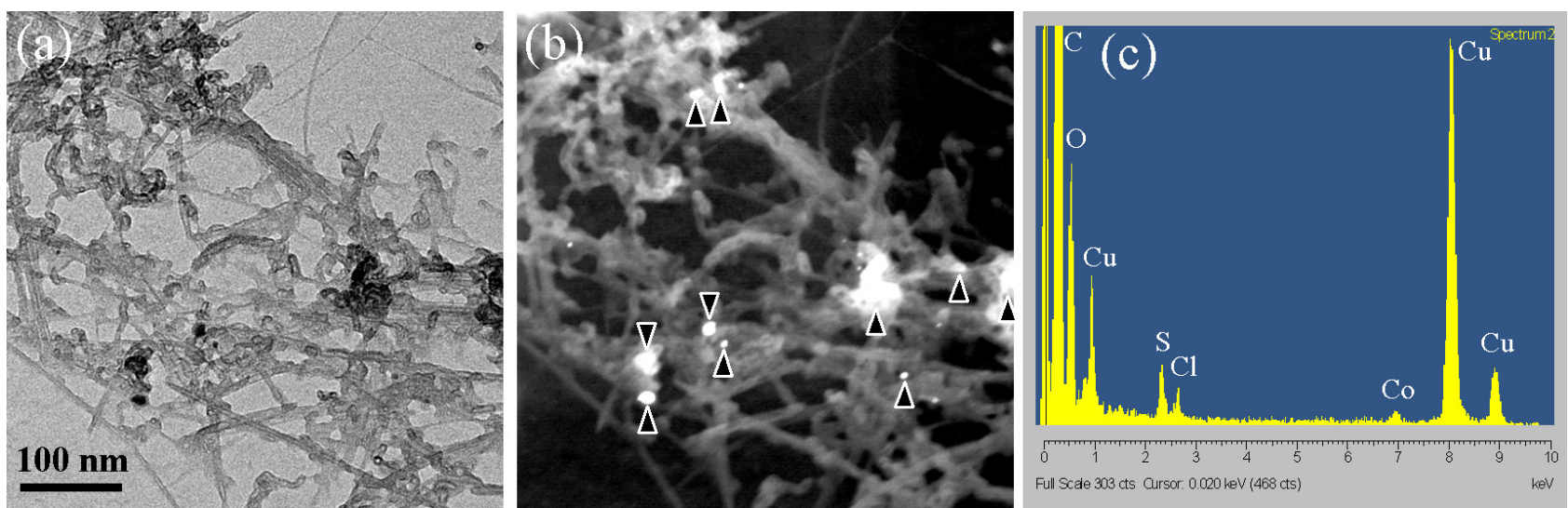

Fig. 1. (a) TEM image; (b) Z-contrast image showing the coatings on the CNT surface; (c) EDS spectrum showing the composition.
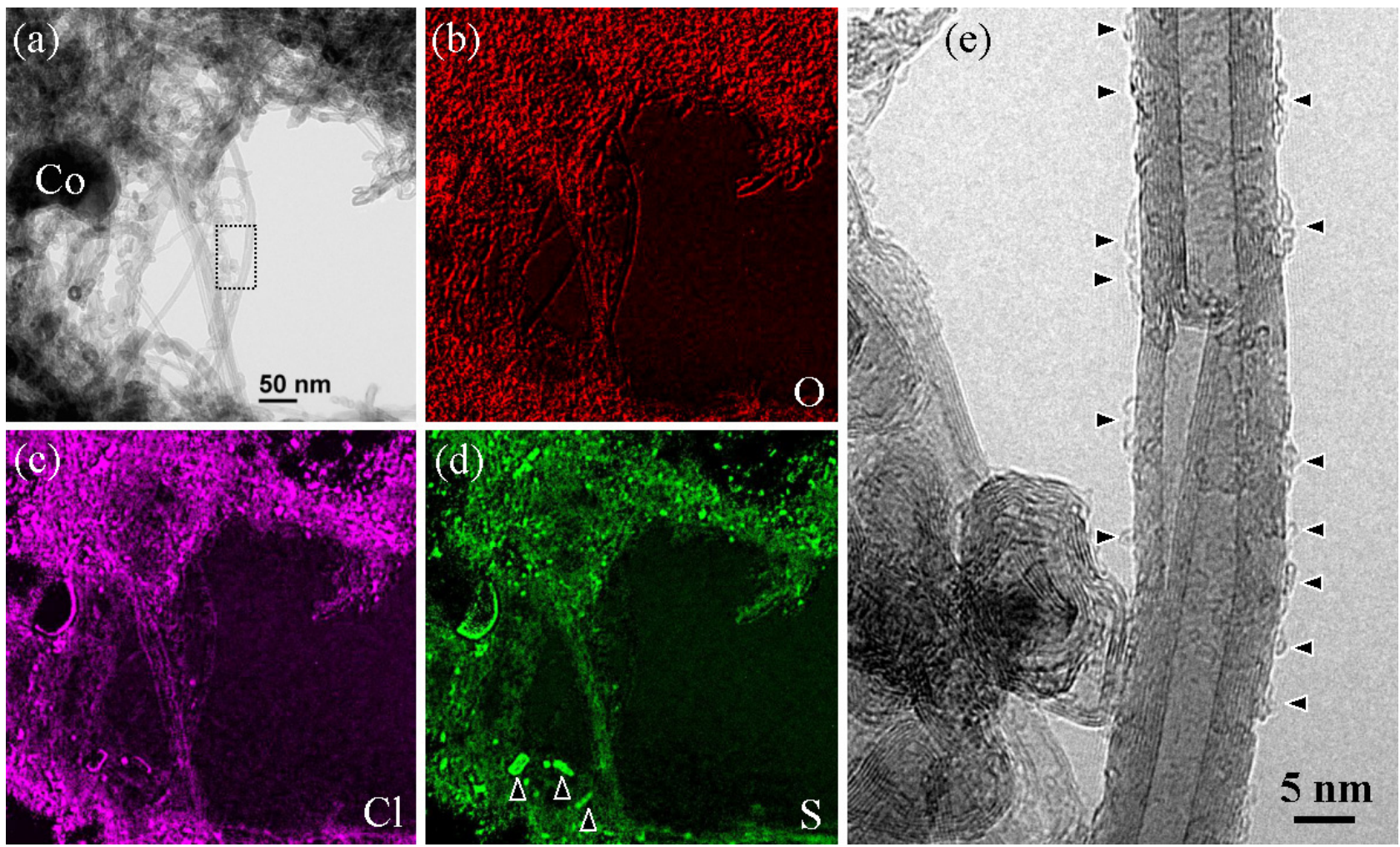

Fig. 2. Zero-loss image (a) and EFTEM elemental maps of $\mathrm{O}, \mathrm{Cl}$ and $\mathrm{S}$ in (b-d) respectively. The framed area in (a) is magnified in (e). Arrowheads indicate the surface coating on this CNT. 